\title{
Reflections: a Daughter's Experience of Parental Cancer and the Beginnings of Un Abrazo Para La Familia ${ }^{\mathrm{Tm}}$
}

\author{
Catherine A. Marshall ${ }^{1}$ (D) \\ Published online: 4 June 2020 \\ (C) American Association for Cancer Education 2020
}

\begin{abstract}
The author describes her impetus and journey in developing Un Abrazo Para La FamiliaTM [Embracing the Family] (Abrazo), 3 hours of cancer information presented in an educational and modular format and designed for low-income informal caregivers who are co-survivors of cancer. A rehabilitation-informed preventive intervention, Abrazo reflects the importance of family, culture, and socioeconomic background in its approach.
\end{abstract}

Keywords Cancer $\cdot$ Caregivers $\cdot$ Cancer knowledge $\cdot$ Poverty $\cdot$ Self-efficacy $\cdot$ Vulnerable and underserved populations

\section{Introduction}

Un Abrazo Para la Familia ${ }^{\mathrm{TM}}$ (Abrazo) was developed as a face-to-face intervention to address the cancer knowledge and self-efficacy needs of low-income caregiving co-survivors of cancer [1], understanding co-survivors to be those affected by the cancer diagnosis of a loved one [2]. The term caregiver applies to family members and friends who provide a range of support services in caring for an individual with an acute or chronic illness [3]. Informal caregivers are predominately female [4] and experience stress at levels that can harm them physically and psychologically [5-7].

While the role of the family in cancer care and the stress associated with a cancer diagnosis has been extensively summarized and documented over the past two decades [6], consensus exists that we still need to better understand the cultural needs of and differences among caregivers [5]. Abrazo was developed with cultural context and other social determinates of health factors in mind [8]. Abrazo is a psychoeducational evidence-based intervention that has demonstrated significant outcomes in cancer knowledge and self-efficacy [2,9-11] for low-income caregiving co-survivors of cancer, as well as for survivors or care recipients [12].

Catherine A. Marshall

marshall@email.arizona.edu

1 Department of Disability and Psychoeducational Studies, The University of Arizona, Tucson, AZ 85721, USA

\section{A Daughter's Experience: Parental Cancer}

Using autoethnography, I first reflected on the role of the family in cancer and within the context of culture and socioeconomic status - my motivation being my experience as a daughter of a man with prostate cancer [13]. My caregiving roles had included (a) helping my father understand and make treatment options, (b) seeking second opinions through a review of medical records and in-person assessment by a specialist, and (c) driving him multiple times from our home in Georgia to treatments provided by specialists at the University of Virginia (956 miles round trip) and the University of South Florida (1148 miles round trip). As with too many other low-income folks [8], my father had been diagnosed with a late-stage cancer and died 2.5 years afterward. I certainly felt that for all my efforts, my "caregiving" was too little, too late.

Subsequent to his death in 2000 , and given my professional background in rehabilitation research, I applied for and was accepted to a 1-year fellowship program beginning in January 2002 with the Cancer, Culture and Literacy (CCL) Institute [14] at the H. Lee Moffitt Cancer Center and Research Institute. According to Meade, "the goal of the Institute was to improve care across the continuum of cancer control by enhancing the skills of researchers responsible for creating multicultural, multilingual, and literacy sensitive interventions and communications for a demographically changing population" (p.4) [14]. 


\section{A Caregiving Experience and the Abrazo Model}

I learned through the CCL Institute that when family members are called upon to participate in making treatment decisions, multiple factors may influence their ability to fully participate. Family members may find that they do not have the health literacy (i.e., cancer-related vocabulary and knowledge), problem-solving, or communication skills to most effectively support their relative, or to address the overarching family challenges that cancer can present. This was my case and may be particularly true of those of us from low-income backgrounds $[8,15]$. Specifically, we struggle with:

1. Health literacy. Words we know may have different meanings in an oncology context-one example from my personal experience as my dad's caregiver-I was told to expect a "bleeding event." I truly had no idea what to expect — an ebola-type bleeding? We may not know how to pronounce words, even common terminology used in oncology - a barrier to asking questions for information or clarification. We may not know how to spell terminology related to cancer treatment so note-taking is a problem.

2. Problem-solving. Low-income people can be very proud people and not accept "handouts" or feel inclined to sit with a social worker to complete applications for free or reduced-price medicines. Working with siblings to determine "who pays" when a low-income parent has cancer can be difficult. Even if a family agrees that a second opinion may be warranted, again, the question of "who pays" can pose a problem - there are not only physician costs but potentially travel costs as well if a specialist is not available locally.

3. Communication skills. As mentioned earlier, speaking in "oncology" can present a unique set of communication circumstances from pronunciation to spelling to comprehension of basic terms. And this is assuming the oncologist and the caregiver speak the same language. Apart from terminology, my experience was that communication was not always appropriate-from an oncologist asking my father in my presence about his sexual function to then suggesting a physical exam before I could scramble out of the room. What might be fine for a wife to hear or see might not be appropriate for a daughter who has accompanied her father to his oncology appointment. Communication may need to be improved not only from the family perspective but from the practitioner perspective as well. Indeed, the diagnosing urologist told me he had a great deal of difficulty giving a cancer diagnosis to his patients.

4. Overarching family challenges. The challenges my family faced were many and, as I learned later, reflective of other low-income families - perhaps especially those uninsured families whose first encounter with the medical establishment brings along a cancer diagnosis. A few of those challenges:

a. My mother was a passive, fearful person and did not accompany my father to any of his medical, and later, oncology appointments - it was left to me, a daughter, and my two sisters to go with him.

b. My sisters typically did not agree on a way forward with my father's treatment- this included whether or not to take him by ambulance to the hospital on the day he died. My older sister, a nurse, wanted my father to die at home - - he did.

c. Processing information about cancer, its causes and its possible treatments, were the ultimate challenge. My mother internalized the cancer prevention message that what you eat might cause cancer-she said once, "I prepared the food - everything he ate for 50 years." My father searched the internet to find information about power lines causing prostate cancer-he had been convinced that the power lines crossing our property had caused his cancer. Whether or not to have chemotherapy in advance of radiation? Whether or not to have brachytherapy? Whether or not or how to pay for expensive medications? Shortly after my father was diagnosed with cancer, he told me that the doctor only spent 10 min with him [13]. We needed more time than that.

After my father's death from prostate cancer, reflecting upon these factors was the impetus that led me, as a researcher, to ask if these experiences were indeed uniquely my own. Did others from low-income families experience the same stress and distress due to their lack of preparedness when facing the cancer diagnosis of a loved one? I wanted to use my rehabilitation research skills, coupled with my personal experience as daughter of a man with prostate cancer, to understand if other low-income families experienced a cancer diagnosis as my family had.

I received a NIH NCI-sponsored Ruth L. Kirschstein National Research Service Award (NRSA) for Individual Senior Fellowship during 2007-2009. The NRSA allowed me to obtain the data needed to develop a psychoeducational, skill-building, and tailored intervention designed to increase the quality of life, including well-being and productivity, of low-income female family members who were providing socioemotional and/or financial support to a cancer survivor relative who had either cervical or breast cancer - this intervention became Abrazo [2, 9-12]. By first researching the needs of local low-income families facing cancer, irrespective of stage, Abrazo was then developed to meet the specific needs of these family members who had reported that they (1) needed more information about cancer, (2) had trouble 
communicating with their provider, and (3) needed information regarding resources available to them [1].

Having obtained my doctorate in rehabilitation at The University of Arizona (UA) in Tucson in 1985 [13], I returned to UA in 2007 via the NRSA to conduct the research leading to the development of Abrazo. I envisioned Abrazo, as a rehabilitation-informed intervention, as a platform for developing psychosocial oncology evidence-based interventions for families affected by cancer that would also serve as community outreach strategies for low-income and underserved populations [8]. "Over the years the philosophy of rehabilitation has remained focused on the belief that all people are unique and possess dignity and worth" (p.192) [16]. Rehabilitation philosophy embraces the importance of involving family when coping with a chronic illness and utilizing group methods for psychoeducational interventions [13]. Rehabilitation principles acknowledge the importance of education, skills-teaching, and strength-based psychosocial support as components of interventions [17].

\section{Benefit Through ABRAZO}

While rehabilitation informed, Abrazo is conceptualized as a preventive intervention [2]. We use the term preventive intervention in line with literature that indicates that informational approaches can prevent the stress, and clinical depression and anxiety associated with the distress of a cancer diagnosis among families facing cancer [18]. The needs that Abrazo addresses include not only providing knowledge and basic understanding about cancer, but also skill development for use of supportive services, using tools needed to understand cancer stage, and skills to address site-specific issues with their oncology team.

Abrazo participants are typically informal family caregiving co-survivors of cancer who provide a range of services [3]. For instance, a caregiver might provide a loved one with support for bathing, eating, preparing meals, and grocery shopping. Further, as per my personal example, a caregiver may provide support by making and driving a loved one to appointments, paying bills, or keeping records.

In chronic illnesses such as cancer, family caregivers are often involved in shared decision-making regarding treatment. Recognition of this shared decision-making requires strategies for upholding the autonomy of the patient while recognizing and respecting family input and contributions [19]. Given the two decades since the turmoil of my father's diagnosis from prostate cancer, and his subsequent death from the disease, it is my lasting hope that Abrazo can continue to benefit others, ensuring everyone's needs are being met in the family touched by cancer [20]. As stated earlier, Abrazo draws from a rehabilitation perspective, to include positive psychology [21,22]. We leave pathology to the cancer and do not search for it among the dynamics impacting family members facing a loved one with cancer.

\section{Conclusion}

In the 20 years since I began to process my father's death, three areas of overlapping clinical, educational, and research advances have informed the continued work of Abrazo: (1) progress toward meeting the needs of low-income people within the context of high-cost cancer treatment, (2) continued understanding of co-survivor impact on survivor well-being, and (3) the ubiquity of technology utilized by families and telemedicine.

The Affordable Care Act (ACA) arrived with the hope of preventive and wellness health care to all [23]. My personal hope became that a cancer diagnosis was then not the first encounter for a low-income family with a health care team. While ACA has now been in place for a decade, we still see that the intersection of race and ethnicity with income status requires focused attention to eliminate disparities [24]. Some would argue that the economic implications of cancer may well trump all other concerns [25]. Financial toxicity is now being discussed in regard to cancer treatment [26, 27].

We have understood that social support can impact survivor outcomes [28]. Researchers continue to look at the implications of social support, for instance, caregiver psychological states upon survivor outcomes - recently adding the dynamic of SES-related stress and finding that caregiver stress would indeed predict depression and anxiety in the survivor [29].

In 2006, we wrote of the promise of telemedicine to allow the underserved, especially those in rural and remote areas, access to health care advocates and to specialized medical knowledge [30]. Importantly, we also noted that telemedicine could be a conduit for providing cultural expertise in an intervention as well. Now, 14 years later, telemedicine is no longer just a promise nor is the technology associated with it. In 2020, most folks have smartphones and are savvy, for instance, with FaceTime, WhatsApp, and Zoom. Families can connect readily through technology. This was not the case in 1998 when my father was diagnosed with prostate cancer. We were concerned with the cost of long-distance calls. Seeing each other meant a flight.

Telehealth has become far more common than we might like during the time of COVID-19. Our comfort in using, and our need to use, the tools available to telemedicine will have forever changed the way we provide information, education, and support. The digitization of Abrazo is underway so that families may access the intervention online if faceto-face is not an option. Thus, whether Abrazo is delivered in person or via some form of telehealth, an embrace, a virtual hug for the low-income family facing cancer, continues to be relevant and needed. 
Acknowledgements Research leading to the development of Un Abrazo Para La Familia (Abrazo) was supported by a Ruth L. Kirschstein National Research Service Award (NRSA) for Individual Senior Fellowship (grant number F33CA117704) from the Department of Health and Human Services' National Institutes of Health and National Cancer Institute awarded to Catherine A. Marshall, PhD, P.I. The NRSA was sponsored by Francisco A. R. Garcia, MD, MPH and co-sponsored by Terry A. Badger, PhD, RN, FAAN; Linda K. Larkey, Ph.D.; and Karen L. Weihs, M.D.

\section{References}

1. Marshall CA, Weihs KL, Larkey LK, Badger TA, Koerner SS, Curran MA, Pedroza R, García FAR (2011) "Like a Mexican wedding": the psychosocial intervention needs of predominately Hispanic low-income female co-survivors of cancer. J Fam Nurs 17(3):380-402

2. Marshall CA et al (2013) Un Abrazo Para La Familia: providing low-income Hispanics with education and skills in coping with breast cancer and caregiving. Psychooncology 22(2):470-474

3. National Cancer Institute (2008) Caring for the caregiver. Journal of Pain \& Palliative Care Pharmacotherapy 22(2):159-164

4. Talley RC, Crews JE (2007) Framing the public health of caregiving. Am J Public Health 97(2):224-228

5. Littleton-Kearney MT, Grady PA (2018) The science of caregiving bringing voices together: summary of National Institute of Nursing Research's 2017 summit. Nurs Outlook 66(2):157-159

6. Northouse LL, Katapodi MC, Schafenacker AM, Weiss D (2012) The impact of caregiving on the psychological well-being of family caregivers and cancer patients. Semin Oncol Nurs 28(4):236-245

7. Kim Y, Carver CS (2019) Unmet needs of family cancer caregivers predict quality of life in long-term cancer survivorship. J Cancer Surviv 13:749-758

8. Marshall CA, Larkey LK, Curran MA, Weihs KL, Badger TA, Armin J, García F (2011) Considerations of culture and social class for families facing cancer: the need for a new model for health promotion and psychosocial intervention. Fam Syst Health 29(2): 81-94

9. Marshall CA, Curran MA, Brownmiller G, Solarte A, Armin J, Hamann HA, Crist JD, Niemelä M, Badger TA, Weihs KL (2018) Oregon's Familias en Acción replicates benefits for underserved cancer co-survivors through Un Abrazo Para la Familia. Psycho-Oncology 27:2405-2411

10. Marshall CA, Curran MA, Koerner SS, Kroll T, Hickman AC, García F (2014) Un Abrazo Para La Familia: an evidenced-based rehabilitation approach in providing cancer education to low-SES Hispanic co-survivors. J Cancer Educ 29(4):626-633

11. Marshall CA, Curran MA, Koerner SS, Weihs KL, Hickman AC, García FAR (2013) Information and support for co-survivors during or after cancer treatment: consideration of Un Abrazo Para la Familia as a model for family-focused intervention in cancer rehabilitation. WORK: A Journal of Prevention, Assessment, and Rehabilitation 46:395-405

12. Marshall CA et al (2020) The evolution of Un Abrazo Para La Familia: implications for survivors of cancer. J Cancer Educ

13. Marshall CA (2008) Family and culture: using autoethnography to inform rehabilitation practice with cancer survivors. J Appl Rehabil Couns 39(1):9-19
14. Meade CD (2005) Cancer, culture and literacy: critical next steps in improving care for diverse populations. SAGE Publications Sage CA, Los Angeles

15. Freeman HP (2004) Poverty, culture, and social injustice: determinants of cancer disparities. CA Cancer J Clin 54(2):72-77

16. Tarvydas V, Addy A, Fleming A (2010) Reconciling evidencedbased research practice with rehabilitation philosophy, ethics and practice: from dichotomy to dialectic. Rehabilitation Research, Policy, and Education 24(3/4):191

17. Marshall CA (1989) Skill teaching in rehabilitation counselor education. Rehabilitation Education 3:9-26

18. Badger TA, Segrin C, Figueredo AJ, Harrington J, Sheppard K, Passalacqua S, Pasvogel A, Bishop M (2011) Psychosocial interventions to improve quality of life in prostate cancer survivors and their intimate or family partners. Qual Life Res 20(6):833-844

19. Laidsaar-Powell R, Butow P, Bu S, Charles C, Gafni A, Fisher A, Juraskova I (2016) Family involvement in cancer treatment decision-making: a qualitative study of patient, family, and clinician attitudes and experiences. Patient Educ Couns 99(7):1146-1155

20. Niemelä M, Marshall CA, Kroll T, Curran M, Koerner SS, Räsänen S, García F (2016) A call to action in public health: the need for family-focused preventive interventions with cancer co-survivors. Am J Public Health 106(8):1381-1387

21. Seligman ME, Csikszentmihalyi M (2014) Positive psychology: an introduction. In: Flow and the foundations of positive psychology. Springer, pp 279-298

22. Casellas-Grau A, Font A, Vives J (2014) Positive psychology interventions in breast cancer. A systematic review. PsychoOncology 23(1):9-19

23. Koh HK, Sebelius KG (2010) Promoting prevention through the affordable care act. N Engl J Med 363(14):1296-1299

24. Ell K, Aranda MP, Wu S, Oh H, Lee PJ, Guterman J (2017) Promotora assisted depression and self-care management among predominantly Latinos with concurrent chronic illness: safety net care system clinical trial results. Contemporary Clinical Trials 61:1-9

25. Mosavel M, Sanders K (2011) Needs of low-income African American Cancer survivors: multifaceted and practical. J Cancer Educ 26(4):717-723

26. Herman PM (2018) The problematic economics of integrative oncology. J Altern Complement Med 24(9-10):1025-1027

27. Gilligan AM et al (2018) Death or debt? National estimates of financial toxicity in persons with newly-diagnosed cancer. Am J Med 131(10):1187-1199. e5

28. Weihs KL, Simmens SJ, Mizrahi J, Enright TM, Hunt ME, Siegel RS (2005) Dependable social relationships predict overall survival in stages II and III breast carcinoma patients. J Psychosom Res 59(5):299-306

29. Segrin C, Badger TA, Sikorskii A, Crane TE, Pace TWW (2018) A dyadic analysis of stress processes in Latinas with breast cancer and their family caregivers. Psycho-oncology 27(3):838-846

30. Marshall CA et al (2006) Considering class, culture, and access in rehabilitation intervention and research. In: Hagglund K, Heinemann A (eds) Handbook of applied disability and rehabilitation research. Springer, New York, pp 25-44

Publisher's Note Springer Nature remains neutral with regard to jurisdictional claims in published maps and institutional affiliations. 